TRANSACTIONS OF THE

AMERICAN MATHEMATICAL SOCIETY

Volume 359, Number 1, January 2007, Pages 143-164

S 0002-9947(06)04095-5

Article electronically published on August 24, 2006

\title{
MULTI-VALUED GRAPHS IN EMBEDDED CONSTANT MEAN CURVATURE DISKS
}

\author{
GIUSEPPE TINAGLIA
}

\begin{abstract}
In this paper we prove that an embedded constant mean curvature disk with Gaussian curvature large at a point contains a multi-valued graph around that point on the scale of $|A|^{2}$. This generalizes Colding and Minicozzi's result for minimal surfaces.
\end{abstract}

\section{INTRODUCTION}

In this paper we prove that an embedded and simply connected constant mean curvature (CMC) surface with Gaussian curvature large at a point contains a multivalued graph around that point on the scale of $|A|^{2}$, where $|A|^{2}$ is the norm squared of the second fundamental form. More precisely, our main result is the following:

Theorem 0.1. Given $N \in \mathbb{Z}_{+}, \omega>1$ and $\varepsilon>0$, there exist $C=C(N, \omega, \varepsilon)>0$, $H>0$ and $\bar{l}>1$. So, let $\Sigma \subset \mathbb{R}^{3}$ be an embedded and simply connected constant mean curvature equal to an $h$-surface. If $|h|<\frac{H}{r_{0}}$ and

$$
\sup _{\Sigma \cap B_{r_{0} \bar{l}}(0)}|A|^{2} \leq 4 C^{2} r_{0}^{-2} \text { and }|A|^{2}(0)=C^{2} r_{0}^{-2}
$$

for some $r_{0}>0$, then $\Sigma$ (after a rotation) contains an $N$-valued graph $\Sigma_{g} \subset \Sigma$ over $D_{\omega \bar{R}} \backslash D_{\bar{R}}$ where $\bar{R}<\frac{r_{0}}{\omega}$ (with gradient $\leq \varepsilon$ and dist $\left._{\Sigma}\left(0, \Sigma_{g}\right) \leq 4 \bar{R}\right)$.

Roughly speaking, to contain a multi-valued graph (Definition 2.2 in this paper) means that locally the surface spirals like a helicoid; see Figure 1. The helicoid is a minimal surface parameterized in the following way:

$$
(s \sin t, s \cos t, t) \quad \text { where }(s, t) \in \mathbb{R}^{2} .
$$

Our result is a generalization of Colding and Minicozzi's result [4, Theorem 0.4] (Theorem 2.1 in this paper) which is a key ingredient in their series of papers [3, 4, 5, 6] that dealt with the structure of embedded minimal disks. We prove that under equivalent local conditions an embedded CMC disk contains a multi-valued graph as well. For a minimal surface, Colding and Minicozzi were able to extend the multi-valued graph that forms locally, all the way up to the boundary [3. It is not known if the same can be done for CMC surfaces. This result can be seen as a first step towards a classification of singularities for sequences of embedded CMC disks; indeed, much more needs to be done in this direction.

The proof is by contradiction using a new compactness argument that does not require a bound on the area. The idea is the following: Assuming that Theorem 0.1

Received by the editors October 4, 2004.

2000 Mathematics Subject Classification. Primary 53A10.

(C)2006 American Mathematical Society Reverts to public domain 28 years from publication 


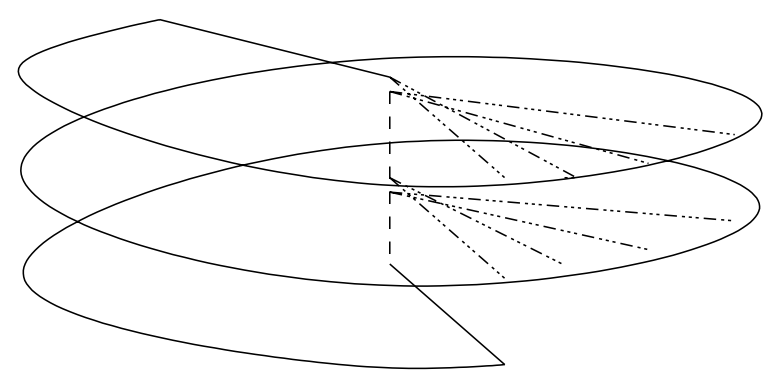

Figure 1. Half of the the helicoids

is false, we build a sequence $\Sigma_{n}$ of embedded CMC disks where each disk satisfies the hypotheses of the theorem with $C$ fixed large and $H=\frac{1}{n}$ but does not contain an $N$-valued graph. We prove that $\Sigma_{n}$ converges $C^{2}$ to a minimal surface $\Sigma_{\infty}$ which contains an $N$-valued graph.

Definition 0.2. A sequence $\Sigma_{n}$ of surfaces converges to a surface $\Sigma_{\infty}$ in the $C^{k}$ topology if at any point $p \in \Sigma_{\infty}$ each $\Sigma_{n}$ is locally (near $p$ ) a graph over the tangent space $T_{p} \Sigma_{\infty}$ and the graph of $\Sigma_{n}$ converges to the graph of $\Sigma_{\infty}$ in the usual $C^{k}$ topology.

We will also consider sequences that converge with multiplicity. This means that we allow more than one graph in the previous definition.

Essentially we show that $\Sigma_{n}$ comes as close as we want to its limit and that the limit is an embedded minimal disk which contains an $N$-valued graph because of Theorem 0.4 in 4 4; therefore, so do the CMC surfaces. To create the $N$-valued graph in the CMC sequence we basically push the multi-valued graph from the minimal surface onto $\Sigma_{n}$. This contradiction proves the theorem.

The difficult part of the proof is to show that the limit is both an embedded surface and simply connected and not, for instance, a minimal lamination or a minimal surface which is not simply connected. Some sort of $C^{2}$ convergence follows in a standard way from the bound on the curvature and trivially, since we are assuming that the mean curvature goes to zero, the limit is a minimal object. To assure that the limit is embedded and simply connected we need a uniform upper bound on the number of graphs in Definition 0.2. We investigate the strong stability for a constant mean curvature surface to find out when a CMC surface, which is already a critical point for a certain area functional, is an actual minimum.

First, we prove that, under certain conditions, if two CMC surfaces are close and disjoint, they are almost-stable.

Second, we rule out the possibility that $\Sigma_{n}$ contains a large, almost-stable domain ("almost a minimum"), for $n$ large.

Third, we show that if there is not a uniform upper bound on the number of pieces, then a large piece of $\Sigma_{n}$ is a graph over another piece, creating a large almost-stable domain and giving the contradiction.

Once the uniform upper bound on the number of pieces is obtained, the convergence to an embedded minimal surface follows. We have to use some topological results to prove that the limit minimal surface is simply connected.

In the first section we provide a short overview of constant mean curvature surfaces. In the second section we describe what a multi-valued graph is and go 
over the hypotheses of the main result. We also take a closer look at the proof. In the third section we deal with the $\delta$-stability for CMC surfaces, and give a criteria to find $\delta$-stable domains in CMC surfaces. In the fourth section we show how, because of the upper bound on $|A|^{2}$, our CMC disk is "uniformly locally flat" and we give a criteria to find large $\delta$-stable domains. In the fifth section we prove that a large $\delta$-stable domain cannot be contained in $\Sigma_{n}$ for $n$ large and how this gives an upper bound on the number of graphs. In the sixth section we prove that the limit is an embedded minimal disk and from that we build a multi-valued graph in $\Sigma_{n}$.

We actually prove the result when $r_{0}$ in Theorem 0.1 is fixed and equal to one. The main result will follow by rescaling and in Appendix B we describe the rescaling argument for constant mean curvature graphs. In Appendix A we provide examples of CMC surfaces containing arbitrary large multi-valued graphs.

\section{Constant mean Curvature surfaces}

This section is a short review of general properties of CMC surfaces.

Let $\Sigma \subset \mathbb{R}^{3}$ be a 2-dimensional smooth orientable surface (possibly with boundary) with unit normal $N_{\Sigma}$. Given a function $\phi$ in the space $C_{0}^{\infty}(\Sigma)$ of infinitely differentiable (i.e., smooth), compactly supported functions on $\Sigma$, consider the oneparameter variation,

$$
\Sigma_{t, \phi}=\left\{x+t \phi(x) N_{\Sigma}(x) \mid x \in \Sigma\right\},
$$

and let $A(t)$ be the area functional,

$$
A(t)=\operatorname{Area}\left(\Sigma_{t, \phi}\right)
$$

The so-called first variation formula of area is the equation (integration is with respect to $d$-area)

$$
A^{\prime}(0)=\int_{\Sigma} \phi H
$$

where $H$ is the mean curvature of $\Sigma$. When $H$ is constant, the surface is said to be a constant mean curvature (CMC) surface [14] and it is a critical point for the area functional restricted to those variations which preserve the enclosed volume; in other words, $\phi$ must satisfy the condition,

$$
\int_{\Sigma} \phi=0
$$

In general, if $\Sigma$ is given as graph of a function $u$, then

$$
H=\operatorname{div}\left(\frac{\nabla u}{\sqrt{1+|\nabla u|^{2}}}\right) \text {. }
$$

Therefore, when $H$ is constant, $u$ satisfies a quasi-linear differential equation. In the particular case where the mean curvature $H$ is identically zero the surface $\Sigma$ is said to be a minimal surface [16, 2]. Concrete examples of constant mean curvature surfaces are spheres, cylinders and Delauney surfaces.

Let $u_{1}, u_{2}$ be CMC graphs over $D_{r}(0)$ and assume that they have the same constant mean curvature $\left(H_{u_{1}}=H_{u_{2}}\right)$, the same orientation $\left(\left\langle N_{1}, N_{2}\right\rangle>0\right)$, and that $u_{1}-u_{2}>0$. Then [2, Lemma 1.17] $u_{1}-u_{2}=v$ is a positive solution of

$$
\operatorname{div} A_{i j} \nabla v+b \nabla v=0
$$




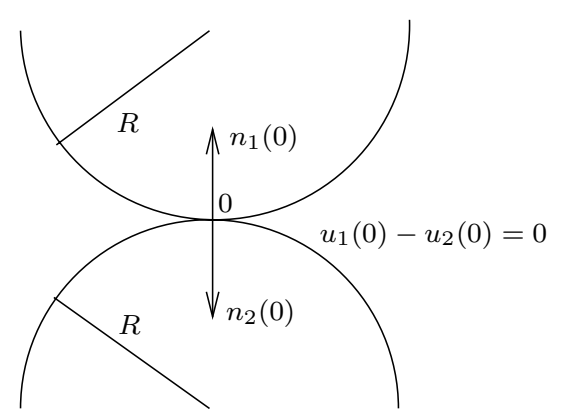

FiguRE 2.

where $A_{i j}$ and $b$ depend on $\nabla u_{1}$ and $\nabla u_{2}$. Moreover, if $\left|\nabla u_{1}\right|$ and $\left|\nabla u_{2}\right|$ are sufficiently small, we have the Harnack type inequality

$$
\sup _{B_{\frac{r}{2}}(0)}\left(u_{1}-u_{2}\right) \leq C_{0}\left(u_{1}(0)-u_{2}(0)\right) .
$$

Notice from Figure 2 that the condition $\left\langle N_{1}, N_{2}\right\rangle>0$ on the orientation is necessary. As shown in Figure 2 the two spherical caps have the same constant mean curvature since they have the same radius. However, even if $u_{1}(0)-u_{2}(0)=0$, it is clear that $\sup \left(u_{1}-u_{2}\right)>0$ in any neighborhood of 0 and therefore that (1.4) does not follow. In general, let $k_{1}, k_{2}$ be the principal curvatures on $\Sigma$, then $H=k_{1}+k_{2}$; $|A|^{2}=k_{1}^{2}+k_{2}^{2}$ is the norm squared of the second fundamental form. Since the Gaussian curvature $K_{\Sigma}$ is equal to the product of the principal curvatures $k_{1} k_{2}$, we have the Gauss equation, that is,

$$
H^{2}=|A|^{2}+2 K_{\Sigma}
$$

From (1.5) it is clear why when $H$ is constant, in particular, when it is small and even better when it is zero, talking about the Gaussian curvature or talking about the norm of the second fundamental form squared is almost equivalent.

\section{Multi-VAlued GRaphs in CMC SuRfaces}

In this section we discuss the result and explain the necessity of the hypotheses. We also take a closer look at how the proof goes.

The following is what Colding and Minicozzi proved.

Theorem 2.1 (4, Theorem 0.4]). Given $N \in \mathbb{Z}_{+}, \omega>1$ and $\varepsilon>0$, there exist $C=C(N, \omega, \varepsilon)>0$. So, let $0 \in \Sigma \subset B_{R} \subset \mathbb{R}^{3}$ be an embedded minimal disk such that $\partial \Sigma \subset B_{R}$. If

$$
\sup _{\Sigma \cap B_{r_{0}}}|A|^{2} \leq 4 C^{2} r_{0}^{-2} \text { and }|A|^{2}(0)=C^{2} r_{0}^{-2}
$$

for some $0<r_{0}<R$, then there exists $\bar{R}<\frac{r_{0}}{\omega}$ and (after a rotation) an $N$-valued graph $\Sigma_{g} \subset \Sigma$ over $D_{\omega \bar{R}} \backslash D_{\bar{R}}$ with gradient $\leq \varepsilon$ and $\operatorname{dist}_{\Sigma}\left(0, \Sigma_{g}\right) \leq 4 \bar{R}$.

Definition 2.2 (Multigraph). Let $D_{r}$ be the disk in the plane centered at the origin and of radius $r$ and let $\mathcal{P}$ be the universal cover of the punctured plane $\mathbb{C} \backslash 0$ with global coordinates $(\rho, \theta)$ so $\rho>0$ and $\theta \in \mathbb{R}$. An $N$-valued graph of a function $u$ on the annulus $D_{s} \backslash D_{r}$ is a single-valued graph over $\{(\rho, \theta)|r \leq \rho \leq s,| \theta \mid \leq N \pi\}$. 


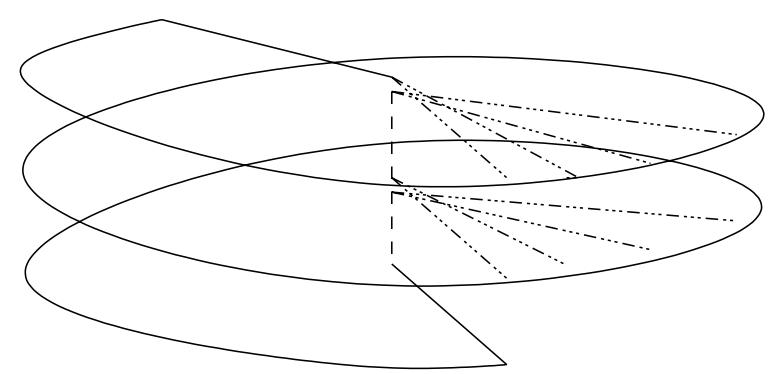

Figure 3. Half of the the helicoids

When dealing with multi-valued graphs, the surface to keep in mind is the helicoid; see Figure 3. A parametrization of the helicoid that illustrates the existence of such an $N$-valued graph is the following:

$$
(s \sin t, s \cos t, t) \quad \text { where }(s, t) \in \mathbb{R}^{2} .
$$

It is easy to see that it contains the $N$-valued graph $\phi$ defined by

$$
\phi(\rho, \theta)=\theta \quad \text { where }(\rho, \theta) \in \mathbb{R}^{+} \backslash 0 \times[-N \pi, N \pi] .
$$

In fact, the helicoid is a minimal surface. In Appendix A we provide examples of CMC surfaces containing arbitrary large multi-valued graphs.

What we are about to prove is not exactly Theorem 0.1. We prove the result when $r_{0}$ in Theorem 0.1 is equal to one and hence the curvature is bounded in a ball of radius $\bar{l}$. We will discuss and determine $\bar{l}$ in Section 5. Theorem 0.1 will follow by rescaling and we will describe the rescaling argument in Appendix B.

This is the new statement:

Theorem 2.3. For each $N \in \mathbb{Z}_{+}, \omega>1$ and $\varepsilon>0$ there exist $H>0, C(N, \omega, \varepsilon)>$ 0 and $\bar{l}>1$. So, let $0 \in \Sigma \subset B_{\bar{l}}(0) \subset \mathbb{R}^{3}$ be an embedded and simply connected constant mean curvature surface equal to $h$ (embedded CMC disk) such that $|h| \leq H$ and $\partial \Sigma \subset \partial B_{\bar{l}}(0)$. If

$$
\sup _{\Sigma \cap B_{\bar{l}}(0)}|A|^{2} \leq 4 C^{2} \text { and }|A|^{2}(0)=C^{2},
$$

then there exists $\bar{R}<\frac{1}{\omega}$ and (after a rotation) an $N$-valued graph $\Sigma_{g} \subset \Sigma$ over $D_{\omega \bar{R}} \backslash D_{\bar{R}}$ (with gradient $\leq \varepsilon$ and $\left.\operatorname{dist}_{\Sigma}\left(0, \Sigma_{g}\right) \leq 4 \bar{R}\right)$.

The constant $C(N, \omega, \varepsilon)$ is essentially the same constant that Colding and Minicozzi used.

We can only prove that a multi-valued graph exists substantially far away from the boundary, that is in a ball of radius one while the boundary of the surface is contained in the boundary of a ball of radius $\bar{l}>1$. For a minimal surface, Colding and Minicozzi were able to extend the multi-valued graph that forms locally, all the way up to the boundary [3]. It is not known if the same can be done for CMC surfaces.

Thanks to the upper bound on the second fundamental form, the surface is "uniformly locally flat" and the $C^{2}$ convergence follows. Moreover, $\sup _{\Sigma \cap B_{\bar{l}}(0)}|A|^{2} \leq$ $4 C^{2}$ together with the Gauss equation (1.5) gives a lower bound for the Gaussian 
curvature,

$$
K_{\Sigma} \geq-4 C^{2}=2 G
$$

This lower bound implies an upper bound on the area of the intrinsic balls, Theorem 5.2 .

What follows is a short sketch of the proof. The proof is by contradiction. Assuming that Theorem 2.3 is false we have the following:

Given $C(N, \omega, \varepsilon)$ as in Theorem 2.1, for any $h>0$ there exists an embedded and simply connected constant mean curvature surface $\Sigma_{h}$ equal to $h$ that does not contain an $N$-valued graph $\Sigma_{g} \subset \Sigma$ over $D_{\omega \bar{R}} \backslash D_{\bar{R}}$ for any $\bar{R}<\frac{1}{\omega}$, but such that

$$
0 \in \Sigma \subset B_{\bar{l}}(0) \subset \mathbb{R}^{3}, \partial \Sigma \subset \partial B_{\bar{l}}(0) \text { and } \sup _{\Sigma \cap B_{\bar{l}}}|A|^{2} \leq 4 C^{2}=4|A|^{2}(0) .
$$

We want to show that this cannot be true. Let us take a sequence of $\Sigma_{n}$ as above with $h=\frac{1}{n}$. The constant mean curvature of $\Sigma_{n}$ goes to zero but none of the elements in the sequence contain an $N$-valued graph. For fixed $\bar{\varepsilon}>0$, we consider a new sequence $\Sigma_{n}^{\prime}$ where $\Sigma_{n}^{\prime}$ is the connected component of $\Sigma_{n} \cap B_{\bar{l}-\bar{\varepsilon}}(0)$ that contains 0 . Given that $|A|^{2}$ is bounded and we are slightly away from the boundary, there exists $r>0$. So, $\Sigma_{n}^{\prime}$ can be covered by a finite number of balls, $B_{r}\left(x_{i}^{n}\right)$ where $x_{i}^{n} \in \Sigma_{n}^{\prime}$, such that in each ball $\Sigma_{n}^{\prime} \cap B_{r}\left(x_{i}^{n}\right)$ looks like graphs $u_{n}^{j}$ over the tangent plane $T_{x_{i}^{n}} \Sigma_{n}$. The radius $r$ and the number of balls will be independent of $n$. Going to a subsequence, we can assume that $x_{i}^{n}$ converges to a certain $x_{i}$ and that $T_{x_{i}^{n}} \Sigma_{n}$ converges to a certain $T_{x_{i}} \Sigma_{\infty}$. At this point we are able to extract, by using Arzela-Ascoli, a subsequence $u_{n}^{j}$ that converges uniformly to a graph $u_{\infty}^{j}$. These CMC graphs satisfy the following partial differential equation

$$
\frac{1}{n}=\operatorname{div}\left(\frac{\nabla u_{n}^{j}}{\sqrt{1+\left|\nabla u_{n}^{j}\right|^{2}}}\right) .
$$

Therefore, using Schauder theory $\left[9\right.$ and the fact that $\frac{1}{n}$ goes to zero, we can prove that $u_{n}^{j}$ converges $C^{2}$ to $u_{\infty}^{j}$ and that the latter is a minimal graph.

Unfortunately, we need more to prove the global properties required. The limit object contains a multi-valued graph if it is an embedded and simply connected minimal surface. We have not ruled out the possibility that the number $j$ of graphs $u_{n}^{j}$ goes to infinity as $n$ goes to infinity and in the limit that could give an infinite number of minimal graphs. As a consequence the limit would not necessarily be a surface but it could be a lamination. Another possibility is that the limit is not simply connected, for instance, it could be a catenoid; see Figure 4, Rescaling the catenoid in Figure 4 the curvature at $p$ becomes very large and yet the catenoid would not contain a multi-valued graph.

What we show is that the number of graphs is uniformly bounded if we stay substantially away from the boundary. This is because to prove this uniform upperbound on the number of graphs we have to work with large geodesic balls and to assure that they exist, we need to move substantially away from the boundary. We need to be working in the unit ball and keep the boundary of the surface on a substantially bigger ball. More precisely, we build another subsequence $\Sigma_{n}^{1}$ where $\Sigma_{n}^{1}$ is the connected component of $\Sigma_{n} \cap B_{1}(0)$ that contains 0; see Figure $5 . \quad \Sigma_{n}^{1}$ is also simply connected. If it was not simply connected, there would exist $B_{r}(0)$, 


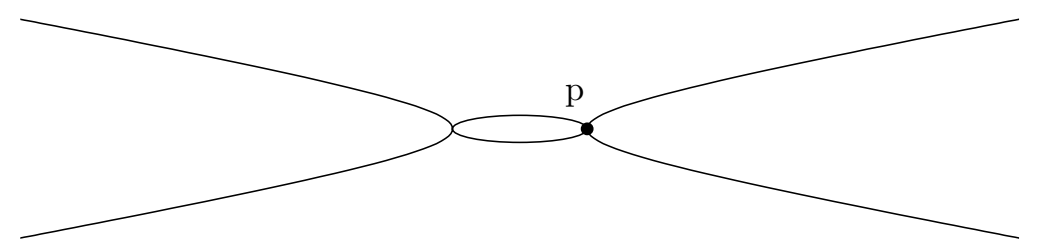

Figure 4. Catenoid

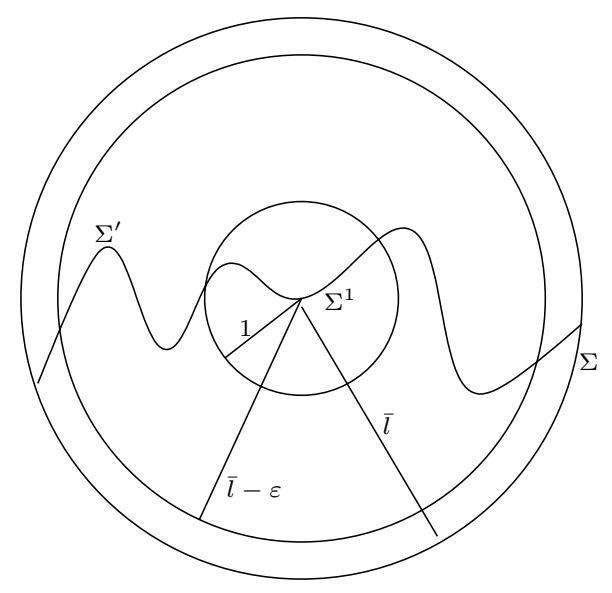

FIGURE 5.

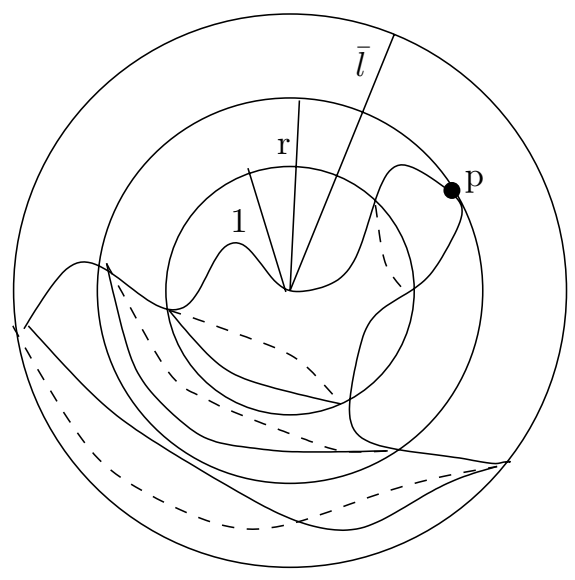

Figure 6.

$1<r<\bar{l}$, such that $\Sigma_{n}$ is tangent to $\partial B_{r}(0)$ and locally inside $B_{r}(0)$. This is a contradiction for $H(n)<\frac{1}{2 l}$; see Figure [6. If we restrict our attention to $\Sigma_{n}^{1}$ we have a uniform upper bound on the number of graphs and it follows that $\Sigma_{n}^{1}$, not the whole $\Sigma_{n}$, converges to an embedded minimal disk. Once we have that $\Sigma_{n}^{1}$ converges to an embedded minimal disk, we prove that $\Sigma_{n}^{1}$, and therefore $\Sigma_{n}$, contains a multi-valued graph. 
From now on, even if the results can often be stated more generally, $\Sigma$ will be a CMC surface satisfying the hypotheses of Theorem 2.3. $\Sigma^{\prime}$ will be the connected component of $\Sigma \cap B_{\bar{l}-\bar{\varepsilon}}(0)$ containing $0 . \Sigma^{1}$ will be the connected component of $\Sigma \cap B_{1}(0)$ containing 0 . We will also assume the mean curvature to be as small as we need, in particular, bounded.

\section{3. $\delta$-STABILITY}

This section consists of standard results about CMC surfaces and stability.

Let $A$ be the area functional described in Section [1 we showed that $A^{\prime}(0)=$ $\int_{\Sigma} \phi H$. A computation shows that if $\Sigma$ is a CMC surface, then

$$
A^{\prime \prime}(0)=-\int_{\Sigma} \phi L_{\Sigma} \phi, \quad \text { where } L_{\Sigma} \phi=\Delta_{\Sigma} \phi+|A|^{2} \phi
$$

is the second variational operator. Here $\Delta_{\Sigma}$ is the intrinsic Laplacian on $\Sigma$. A CMC surface $\Sigma$ is said to be (strongly) stable if

$$
A^{\prime \prime}(0) \geq 0 \quad \text { for all } \phi \in C_{0}^{\infty}(\Sigma)
$$

Applying Stokes' theorem to (3.2) shows that $\Sigma$ is stable if and only if

$$
\int_{\Sigma}|A|^{2} \phi^{2} \leq \int_{\Sigma}|\nabla \phi|^{2}, \quad \text { for all } \phi \in C_{0}^{\infty}(\Sigma),
$$

and that allows us to define $\delta$-stability, namely $\Sigma$ is said to be $\delta$-stable if

$$
(1-\delta) \int_{\Sigma}|A|^{2} \phi^{2} \leq \int_{\Sigma}|\nabla \phi|^{2}, \quad \text { for all } \phi \in C_{0}^{\infty}(\Sigma) .
$$

In the following lemma we establish a relation between a CMC surface and a $\mathrm{CMC}$ normal variation of it that does not change the mean curvature.

Lemma 3.1. There exists $\delta_{1}>0$. So, if $\delta<\delta_{1}, \Sigma$ is a CMC surface and $u$ is a positive solution of the CMC graph equation over $\Sigma$ (i.e., $\Sigma^{u}:=\left\{x+u(x) N_{\Sigma}(x) \mid x \in\right.$ $\Sigma\}$ is CMC) such that $\left|H_{\Sigma^{u}}\right|=\left|H_{\Sigma}\right|,\left\langle N_{\Sigma^{u}}, N_{\Sigma}\right\rangle \geq 0,|u||A|$ and $|\nabla u| \leq \delta$, then $\triangle u+u|A|^{2}=o\left(\delta^{2}\right)$.

Proof. In general,

$$
H_{\Sigma^{u}}=H_{\Sigma}+\frac{1}{2}\left(\triangle u+u|A|^{2}\right)+o\left(|u|^{2},|\nabla u|^{2}\right) .
$$

The condition $\left\langle N_{\Sigma^{u}}, N_{\Sigma}\right\rangle \geq 0$ is a condition on the orientation that implies $H_{\Sigma^{u}}=$ $H_{\Sigma}$ and the lemma follows.

The existence of a positive solution of $L u=0$ where $L$ is $\Delta+|A|^{2}$ would imply $A^{\prime \prime}(0) \geq 0$ for all $\phi \in C_{0}^{\infty}(\Sigma)$. In the following lemma we show that if there exists a positive function $u$ which is "almost" a solution, then $A^{\prime \prime}(0)$ is "almost" non-negative for all $\phi \in C_{0}^{\infty}(\Sigma)$, that is, almost-stable.

Lemma 3.2. Let $\Omega$ be a domain and let $u$ be a positive function in $C^{2}(\Omega)$ such that

$$
\Delta u \leq-(1-\delta)|A|^{2} u
$$

Then $\Omega$ is $\delta$-stable. 
Proof. Set $w=\log u$ and let $\Phi$ be any compactly supported function on $\Omega$. We have

$$
\operatorname{div}(\nabla w)=\operatorname{div}\left(\frac{\nabla u}{u}\right)=\frac{\triangle u}{u}-\frac{|\nabla u|^{2}}{u^{2}}=\frac{\triangle u}{u}-|\nabla w|^{2} .
$$

Applying Stokes theorem to $\operatorname{div}\left(\Phi^{2} \nabla w\right)$ gives

$$
0=\int \operatorname{div}\left(\Phi^{2} \nabla w\right)=\int \Phi^{2} \Delta w+\int\left\langle\nabla \Phi^{2}, \nabla w\right\rangle .
$$

Using Cauchy-Schwarz and the absorbing inequality gives

$$
\int\left\langle\nabla \Phi^{2}, \nabla w\right\rangle \leq \int|\nabla \Phi|^{2}+\int \Phi^{2}|\nabla w|^{2} .
$$

Eventually,

$$
\int\left(-\frac{\triangle u}{u}+|\nabla w|^{2}\right) \Phi^{2} \leq \int|\nabla \Phi|^{2}+\int \Phi^{2}|\nabla w|^{2} .
$$

Applying (3.4) we get

$$
(1-\delta) \int_{\Sigma}|A|^{2} \phi^{2} \leq \int_{\Sigma}|\nabla \phi|^{2}
$$

Lemma 3.1 and Lemma 3.2 give a first criteria to find almost stable domains in a constant mean curvature surface.

Corollary 3.3. There exists $\delta_{3}>0$. So, if $\delta<\delta_{3}, \Sigma$ is a CMC surface and $u$ is a positive solution of the CMC graph equation over $\Sigma$ such that $\left|H_{\Sigma^{u}}\right|=\left|H_{\Sigma}\right|$, $\left\langle N_{\Sigma^{u}}, N_{\Sigma}\right\rangle \geq 0,|u||A|$ and $|\nabla u| \leq \delta$, then $\Sigma$ is $\delta$-stable.

\section{THE UPPER BOUND ON $|A|^{2}$}

In this section we use the upper bound on $|A|^{2}$ to generalize some standard local results regarding CMC surfaces. We prove a criteria to find large pieces of $\Sigma^{\prime}$ which are graph over other pieces, creating large almost stable CMC domains.

Let us define

$$
\Sigma_{x, R} \text { as the component of } B_{R}(x) \cap \Sigma \text { that contains } x,
$$

$$
\mathcal{B}_{R}(x):=\left\{y \in \Sigma \text { such that } \operatorname{dist}_{\Sigma}(y, x)<R\right\},
$$

i.e., the geodesic ball of radius $R$ centered at $x$,

$$
\mathcal{D}_{r}(x):=\left\{x^{\prime} \in T_{x} \Sigma \text { such that }\left|x-x^{\prime}\right|<r\right\} .
$$

In what follows we will explain why in a CMC surface with bounded $|A|^{2}$ everything looks graphical - what we have been calling "uniformly locally flat." Integrating $\left|\nabla \operatorname{dist}_{S^{2}}(\mathbf{n}(x), \mathbf{n})\right| \leq|A|$ on geodesics gives

$$
\sup _{x^{\prime} \in \mathcal{B}_{s}(x)} \operatorname{dist}_{S^{2}}(\mathbf{n}(x), \mathbf{n}) \leq s \sup _{\mathcal{B}_{s}(x)}|A| .
$$

By (4.4), we can choose $0<\rho<\frac{1}{4}$. So, if $\mathcal{B}_{2 s}(x) \subset \Sigma, s \sup _{\mathcal{B}_{s}(x)}|A| \leq 4 \rho_{2}$, and $t \leq s$, then the component $\Sigma_{x, t}$ of $B_{t}(x) \cap \Sigma$ with $x \in \Sigma_{x, t}$ is a graph over $T_{x} \Sigma$ with gradient $\leq \frac{t}{s}$ and

$$
1 \geq \inf _{x^{\prime} \in \mathcal{B}_{2} s(x)} \frac{\left|x^{\prime}-x\right|}{\operatorname{dist}_{\Sigma}\left(x, x^{\prime}\right)}>\frac{9}{10}
$$




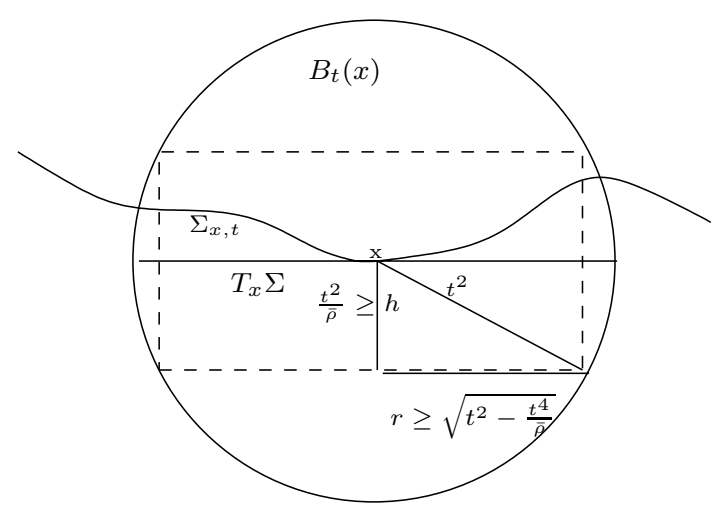

FiguRE 7.

One consequence is that if $t \leq s$ and we translate $T_{x} \Sigma$ so that $x \in T_{x} \Sigma$, then

$$
\sup _{x^{\prime} \in \mathcal{B}_{t}(x)}\left|x^{\prime}-T_{x} \Sigma\right| \leq \frac{t^{2}}{s}
$$

As a consequence of (4.4), (4.5), (4.6) and the fact that in this paper we are assuming $\sup _{\Sigma}|A|<C$, we can clearly choose $0<\bar{\rho}<4 \frac{\rho}{C}$. So, given $t<\bar{\rho}$ and $x \in \Sigma$, then

$$
\Sigma_{x, t} \text { is a graph over } T_{x} \Sigma \text { with gradient } \leq \frac{t}{\bar{\rho}} \text { and } 1 \geq \inf _{x^{\prime} \in \mathcal{B}_{2 \bar{\rho}}(x)} \frac{\left|x^{\prime}-x\right|}{\operatorname{dist}_{\Sigma}\left(x, x^{\prime}\right)}>\frac{9}{10} .
$$

This means that, independently on $x, \Sigma_{x, t}$ is a graph over $T_{x} \Sigma$. Moreover, as shown in Figure 7, using the Pythagorean theorem, gives that

$$
\text { the projection of } \Sigma_{x, t} \text { onto } T_{x} \Sigma \text { contains } \mathcal{D} \sqrt{t^{2}-\frac{t^{4}}{\bar{\rho}^{2}}}(x) .
$$

Furthermore, if $y \in B_{t}(x) \cap \Sigma$ and $\operatorname{dist}_{\Sigma}(x, y) \geq 2 t$, then $y$ cannot be in $\Sigma_{x, t}$, otherwise applying (4.5) gives

$$
t \geq|y-x|>\frac{9}{10} \operatorname{dist}_{\Sigma}(x, y) \geq \frac{18}{10} t .
$$

$y$ is in a different component of $B_{t}(x) \cap \Sigma$. After defining an orientation, $y$ is either above or below $\Sigma_{x, t}$. For the same reason we can also add that $\mathcal{B}_{\bar{\rho}}(x) \cap \mathcal{B}_{\bar{\rho}}(y)=\emptyset$.

Corollary 3.3 tells us that under certain conditions regarding the orientation, if a CMC surface is a graph over another CMC surface with the same constant mean curvature, then it is almost stable. We are about to prove some lemmas which tell us when that happens and how large the almost stable domain is. This lemma shows how, if two pieces of $\Sigma$ are close, then they must be graphs over the same plane.

Lemma 4.1. There exists $\alpha_{1}>0$. So, for any $\alpha<\alpha_{1}$ and $x \in \Sigma^{\prime}$, then any component of $B_{\alpha}(x) \cap \Sigma^{\prime}$ is a graph over $T_{x} \Sigma$.

Proof. Let us assume that there exists a component of $B_{\alpha}(x) \cap \Sigma^{\prime}$ which is not a graph over $T_{x} \Sigma$. Then there exists $y \in B_{\alpha}(x) \cap \Sigma^{\prime}$ such that $T_{x} \Sigma \perp T_{y} \Sigma$. If $\alpha_{1}$ is small enough, it is clear from Figure 8 that $\Sigma_{x, \frac{\bar{\rho}}{2}} \cap \Sigma_{y, \frac{\bar{\rho}}{2}} \neq \emptyset$, that is, $y \in \mathcal{B}_{\bar{\rho}}(x)$. How we have chosen $\bar{\rho}$ implies that $y$ must be part of a graph. Notice that we are 


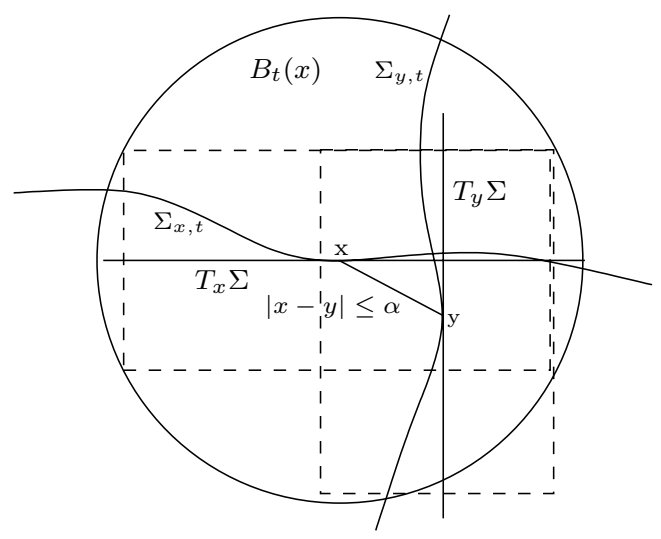

FiguRE 8.

also using the fact that we are slightly away from the boundary. $\Sigma_{x, \frac{\bar{\rho}}{2}} \cap \Sigma_{y, \frac{\bar{\rho}}{2}}$ could be empty if one of the two sets reaches $\partial \Sigma$ before they intersect. How small $\alpha_{1}$ must be will depend also on $\bar{\varepsilon}$.

In particular, it follows that if pieces of $\Sigma^{\prime}$ are very close, then not only are they graphs over the same plane, they are graphs over each other. The idea is that if two graphs are almost flat over two different planes but they cannot intersect, and if the two graphs are close enough, these two planes must have almost the same slope. One of the two graphs can therefore be seen as a graph over the other and this is what the next lemma is about.

Lemma 4.2. There exists $\alpha_{2}>0, C_{1}>0$ and $s>0$. So, let $x, y \in \Sigma^{\prime}$ such that $|x-y| \leq \alpha<\alpha_{2}, d_{\Sigma}(x, y) \geq 2 \alpha$ and $\langle n(x), n(y)\rangle>0$. Then $\mathcal{B}_{s}(y)$ contains a graph $\{z+u(z) n(z)\}$ over a domain containing $\mathcal{B}_{\frac{s}{4}}(x),|\nabla u|+|u| \leq \alpha C_{1}$.

Proof. Assume $\alpha_{2}<\alpha_{1}$. We know from (4.8) that $y$ is in a different component of $B_{\alpha}(x) \cap \Sigma^{\prime}$ and that $\mathcal{B}_{\bar{\rho}}(x) \cap \mathcal{B}_{\bar{\rho}}(y)=\emptyset$. If $\alpha_{2}$ is sufficiently small, it now follows that $\Sigma_{x, \bar{\rho}}$ and $\Sigma_{y, \bar{\rho}}$ contain two graphs over the same plane; the smaller $\alpha_{2}$ is, the bigger the graph is. There exists $s>0$ such that $\mathcal{B}_{s}(x)$ and $\mathcal{B}_{s}(y)$ contain, respectively, a graph $u_{1}$ and $u_{2}$ over $\mathcal{D}_{\frac{s}{2}}(x)$. $\langle n(x), n(y)\rangle>0$ implies that the two intrinsic disks have equal constant mean curvature and (1.4) gives $\sup _{\mathcal{D}_{\frac{s}{4}(x)}}\left|u_{1}-u_{2}\right| \leq \alpha C_{0}$. The function

$$
u(x)=\min \left\{t \in \mathbb{R}_{+} \mid x+t N(x) \in \mathcal{B}_{s}(y)\right\}
$$

is well defined over $B_{\frac{s}{4}}(x)$ and $|\nabla u|+|u| \leq \alpha C_{1}$.

Lemma 3.2 and Lemma 4.2 give a better criteria to find $\delta$-stable domain.

Corollary 4.3. Given $\delta>0$ there exists $\alpha_{3}>0$ and $s>0$. So, let $x, y \in \Sigma^{\prime}$ such that $|x-y| \leq \alpha<\alpha_{3}, d_{\Sigma}(x, y) \geq 2 \alpha$ and $\langle n(x), n(y)\rangle>0$. Then $\mathcal{B}_{\frac{s}{4}}(x)$ is $\delta$-stable.

Proof. Let $\alpha_{3}<\min \left(\frac{\delta}{C_{1}}, \alpha_{2}\right)$ and apply Lemma 3.2 and Lemma 4.2 .

In sum, we have proven that when two points $x, y \in \Sigma^{\prime}$ are close enough to each other (Euclidean distance) and satisfy the condition on the orientation $\langle N(x), N(y)\rangle>0$, then a little neighborhood of each point is $\delta$-stable. We notice 


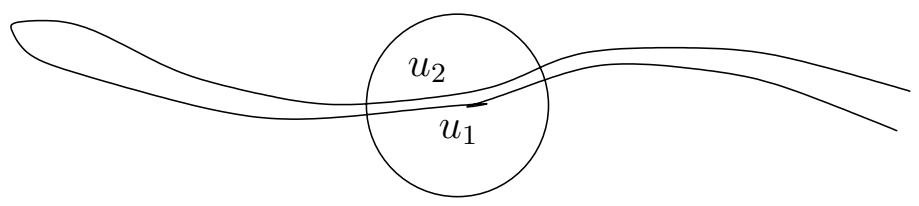

FiguRE 9.

that the closer two pieces are, the smaller $\delta$ is. The next step is to go from a little almost stable domain to a large one.

If we need a very large $\delta$-stable geodesic ball, then first we need the geodesic ball to be contained in $\Sigma$. In order to achieve this we certainly cannot be anywhere in the surface but sufficiently away from its boundary. If we move away from the boundary, as long as the objects we are working with are contained in $\Sigma$, thanks to the Harnack inequality we can find conditions that guarantee the existence of arbitrary large $\delta$-stable domains. This is what we prove in the next lemma and corollaries. In Figure 9 it is shown how if two pieces of $\Sigma^{1}$ are close, then their extensions will have to stay relatively close.

Lemma 4.4. For each $0<l<\bar{l}-(1+\bar{\varepsilon})$ there exist $\alpha_{l}>0$ and $C_{l}>0$. So, given $\alpha<\alpha_{l}$ and $x, y \in \Sigma^{1}$ such that $|x-y| \leq \alpha, d_{\Sigma}(x, y) \geq C_{l}$ and $\langle n(x), n(y)\rangle>0$, then for each $x^{\prime} \in \mathcal{B}_{l}(x)$ there exists $y^{\prime} \in \Sigma^{\prime}$ such that $\left|x^{\prime}-y^{\prime}\right| \leq \alpha_{1}, d_{\Sigma}\left(x^{\prime}, y^{\prime}\right) \geq 2 \alpha_{1}$ and $\left\langle n\left(x^{\prime}\right), n\left(y^{\prime}\right)\right\rangle>0$.

Proof. Fix $N \in \mathbb{N}$ such that $\frac{4 l}{s} \leq N<\frac{4 l}{s}+1, s$ as in Corollary 4.3 and assume $\alpha_{l}<\min \left(\alpha_{2}, \frac{\alpha_{2}}{C_{0}^{N}}\right), C_{0}$ being the constant as in (1.4). Our goal is to find $C_{l}$; note that $\mathcal{B}_{l}(x) \subset \Sigma^{\prime}$. Let $x^{\prime} \in \mathcal{B}_{l}(x)$. Then there exists a geodesic $\gamma(t), t \in[0,1]$, such that $\gamma(0)=x, \gamma(1)=x^{\prime}$ and $\operatorname{length}(\gamma) \leq l$. Fix a partition $\mathcal{Q}$ of $[0,1]$, $\mathcal{Q}=\left\{t_{i} \in[0,1] \mid 0 \leq i \leq T\right\}$, such that

$$
\left\{\begin{array}{l}
t_{0}=0, t_{T}=1, \\
d_{\Sigma}\left(\gamma\left(t_{i}\right)=x_{i}, \gamma\left(t_{i+1}\right)=x_{i+1}\right) \leq \frac{s}{4}, \\
T \leq N .
\end{array}\right.
$$

Since $|x-y| \leq \alpha \leq \alpha_{2}$ and $d_{\Sigma}(x, y) \geq C_{l} \geq 2 \alpha_{2}$, Lemma 4.2 gives $\mathcal{B}_{s}(x)$ and $\mathcal{B}_{s}(y)$ contain, respectively, a graph $u_{1}$ and $u_{2}$ over $\mathcal{D}_{\frac{s}{2}(x)}$ and $\sup _{\mathcal{D}_{\frac{s}{4}(x)}}\left|u_{1}-u_{2}\right| \leq C_{0} \alpha$. Therefore, let $z_{1} \in \mathcal{D}_{\frac{s}{4}}(x)$ such that $x_{1}=u_{1}\left(z_{1}\right)$ and let $y_{1}=u_{2}\left(z_{1}\right)$. Then $\left|x_{1}-y_{1}\right| \leq C_{0} \alpha \leq \alpha_{2}, d_{\Sigma}\left(x_{1}, y_{1}\right) \geq d_{\Sigma}(x, y)-d_{\Sigma}\left(x_{1}, x\right)-d_{\Sigma}\left(y_{1}, y\right) \geq C_{l}-\frac{s}{4}-\frac{5 s}{4} \geq$ $2 \alpha_{2}$ if $C_{l}$ is big enough, and $\left\langle N\left(x_{1}\right), N\left(y_{1}\right)\right\rangle>0$. As long as $d_{\Sigma}\left(x_{i}, y_{i}\right) \geq 2 \alpha_{2}$, we can apply Lemma 4.2. We can repeat this argument $N$ times as long as $C_{l}-N \frac{3 s}{2} \geq$ $2 \alpha_{2}$.

Corollary 4.5. For each $0<l<\bar{l}-(1+\bar{\varepsilon})$ there exist $\alpha_{l}>0$ and $C_{l}>0$. So, given $\alpha<\alpha_{l}$ and $x, y \in \Sigma^{1}$ such that $|x-y| \leq \alpha, d_{\Sigma}(x, y) \geq C_{l}$ and $<n(x), n(y)>>0$, then for each $x^{\prime} \in \mathcal{B}_{l}(x), \mathcal{B}_{t}(y)$ contains a graph $\{z+u(z) n(z)\}$ over a domain containing $\mathcal{B}_{\frac{t}{4}}(x),|\nabla u|+|u| \leq \alpha C_{1}$.

Proof. Apply Lemma 4.4 and Lemma 4.2 .

Corollary 4.6. For each $0<l<\bar{l}-(1+\bar{\epsilon})$ and $\delta>0$ there exist $\alpha_{l, \delta}>0$ and $C_{l}>0$. So, given $\alpha<\alpha_{l, \delta}$ and $x, y \in \Sigma^{1}$ such that $|x-y| \leq \alpha, d_{\Sigma}(x, y) \geq C_{l}$ and $\langle N(x), N(y)\rangle>0$, then $\mathcal{B}_{l}(x)$ is $\delta$-stable. 
Proof. Let $\alpha_{l, \delta}<\min \left(\alpha_{l}, \frac{\delta}{C_{1}}\right)$, according to Corollary 3.3 , we need to find a $u$ which is a positive solution of the CMC graph equation over $\mathcal{B}_{l}(x)$. Corollary 4.5 gives that the latter is true locally. In fact, fix $x_{i} \in \mathcal{B}_{l}(x)$ such that $\mathcal{B}_{s}\left(x_{i}\right)$ and $\mathcal{B}_{\frac{s}{4}}\left(x_{i}\right)$ are both finite coverings for $\mathcal{B}_{l}(x)$. From Lemma 4.4 it follows that $\mathcal{B}_{s}\left(x_{i}\right)$ contains a graph $\left\{z+u_{i}(z) n(z)\right\}$ over a domain containing $\mathcal{B}_{\frac{s}{4}}\left(x_{i}\right),\left|\nabla u_{i}\right|+\left|u_{i}\right| \leq \alpha C_{1}$. The function $u(y):=u_{i}(y)$ if $y \in \mathcal{B}_{\frac{s}{4}}\left(x_{i}\right)$ is a well-defined function over $\mathcal{B}_{l}(x)$ such that $|\nabla u|+|u| \leq \alpha C_{1}<\delta$. Applying Corollary 3.3 gives this corollary.

\section{The NON-EXistence of LARge ALmost Stable Domain AND THE UNIFORM BOUND}

We have seen when it happens that $\Sigma$ contains a large almost stable domain. In this section we show that an almost stable domain cannot be too large. Using these two facts together we prove a uniform bound on the number of graphs.

In order to continue with this proof by contradiction we state the following result by Sirong Zhang [18, Theorem 0.1], and the Bishop Volume Comparison Theorem [17, Theorem 1.3]:

Theorem 5.1. There exists a $C$ such that given any $l>0$ there exists an $h>0$. $S o$, if $\mathcal{B}_{l}(0)$ is a "constant mean curvature equal to $h$ ", $\delta$-stable intrinsic disk with trivial normal bundle, then $\sup _{\mathcal{B}_{\frac{l}{2}}(0)}|A|^{2} \leq \frac{C}{l^{2}}$.

Theorem 5.2 (Bishop Volume Comparison Theorem). Let $M$ be an $n$-dimensional complete Riemannian manifold with $\operatorname{Ric}(M) \geq(n-1) K$. Then for any $x \in M$ and $R>0, \frac{V o l\left(\mathrm{~B}_{R}(x)\right)}{V(K, R)}$ is a non-increasing function in $R$. Hence,

$$
\operatorname{Vol}\left(\mathrm{B}_{R}(x)\right) \leq V(K, R),
$$

where $V(K, R)$ is the volume of the geodesic ball of radius $R$ in the space form $M_{K}$.

Theorem 5.1 can be thought as a generalization of [7, 8] and we will see how it is essentially what determines how big $\bar{l}$ is. Our surface has trivial normal bundle since it is orientable and this will be proved later in Theorem 6.4. Theorem 5.2 gives that for any $x \in \Sigma$

$$
\operatorname{Vol}\left(B_{R}(x)\right) \leq V(G, R), \quad G \text { as in Theorem } 2.1 .
$$

The following proposition uses what we have proved in the previous sections and Theorem 5.1 to show that $\Sigma^{\prime}$ does not contain a large almost stable domain. Roughly speaking, if we take $l$ large and assume that $\mathcal{B}_{l}(x) \subset \Sigma^{1}$ is $\delta$-stable, Theorem 5.1 implies that $\mathcal{B}_{\frac{l}{2}}(x)$ is almost flat. This forces the intrinsic disk to leave the unit ball.

Proposition 5.3. Given $\delta>0$ there exists $l_{\delta}>0$. So, for any $l \geq l_{\delta}$ and $x \in \Sigma^{1}$ if $\mathcal{B}_{l}(x)$ is $\delta$-stable, then it is not contained in $\Sigma^{1}$.

Proof. Let us fix $l_{\delta}>\max \left(\frac{20}{9}, \sqrt{\frac{C}{\rho}}\right)$. Let $C$ be as in Theorem [5.1] and $\rho$ as in (4.5). Being $\mathcal{B}_{l}(x) \delta$-stable, Theorem 5.1 implies

$$
\sup _{\mathcal{B}_{\frac{l}{2}}(x)}|A|^{2} \leq \frac{C}{l^{2}} \leq \rho .
$$




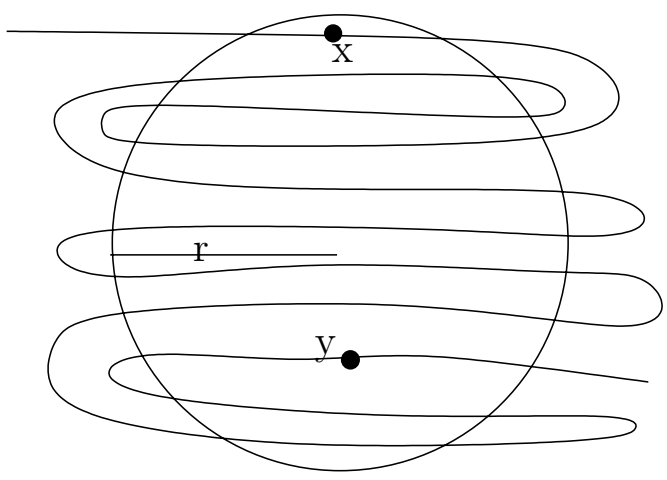

FiguRE 10.

(4.5) implies that

$$
\inf _{\mathcal{B}_{\frac{l}{2}}(x)} \frac{|x-y|}{d_{\Sigma}(x, y)} \geq \frac{9}{10} .
$$

Taking $y \in \mathcal{B}_{\frac{l}{2}}(x)$ such that $d_{\Sigma}(x, y)>\frac{10}{9}$, we have $|x-y|>1$. This proves that $\mathcal{B}_{l}(x)$ is not contained in $\Sigma^{1}$.

We have proved so far that decreasing the Euclidean distance between two points gives a large $\delta$-stable domain as long as we increase their intrinsic distance. In the following lemma we apply the Bishop Volume Comparison Theorem and a lower bound on the area of each piece to prove that the more graphs there are in a small ball the larger the intrinsic distance becomes. This is what Figure 10 illustrates.

Let us fix $\delta, \bar{\varepsilon}>0$ small, let $l_{1}=l_{\delta}$ as given by Proposition 5.3 and let $\bar{l}$ in Theorem 2.3 be equal to $l_{1}+1+\bar{\varepsilon}$. In other words,

$\Sigma^{1}$ does not contain a $\delta$-stable geodesic ball of radius bigger than $l_{1}$.

Let us fix $0<r<\alpha_{l_{1}, \delta}$ where $\alpha_{l_{1}, \delta}$ is taken as in Corollary 4.6. This means that:

For any $x \in \Sigma^{\prime}, B_{r}(x) \cap \Sigma^{\prime}$ consists of graphs over $T_{x} \Sigma$, and also that:

There exists $C_{l_{1}}>0$ such that if $x, y \in B_{r}(x) \cap \Sigma^{1}, d_{\Sigma}(x, y) \geq C_{l_{1}}$ and $\langle N(x), N(y)\rangle>0$, then $\mathcal{B}_{l_{1}}(x)$ is $\delta$-stable.

Given $x \in \Sigma^{1}$ let $n_{x}$ be the number of components of $B_{r}(x) \cap \Sigma^{1}$. The area of each component $\Sigma_{i}^{x}$ could go to zero if they accumulate toward the boundary of the ball. Nonetheless, we have proven that these graphical pieces continue outside the ball, (4.7). Therefore, we have a uniform lower bound on the area of $\Sigma_{i}^{x}$; in other words:

(5.4) There exists $\varepsilon>0$ such that $\operatorname{Area}\left(\Sigma_{i}^{x}\right)>\varepsilon$ independently of $x$ and $i$.

Lemma 5.4. Given $\lambda>0$ there exists $n_{\lambda}>0$. So, if $x \in \Sigma^{1}$ and $n_{x}>n_{\lambda}$, then there exist $y, y^{\prime} \in \mathrm{B}_{r} \cap \Sigma^{1}$ such that $\operatorname{dist}_{\Sigma}\left(y, y^{\prime}\right)>\lambda$ and $\left\langle n(y), n\left(y^{\prime}\right)\right\rangle>0$.

Proof. Theorem 5.2 that is, Bishop Volume Comparison Theorem, gives an upper bound for the area of $\mathcal{B}_{\lambda}(x)$, namely

$$
\operatorname{Area}\left(\mathcal{B}_{\lambda}(x)\right)<V(G, \lambda) \text {. }
$$




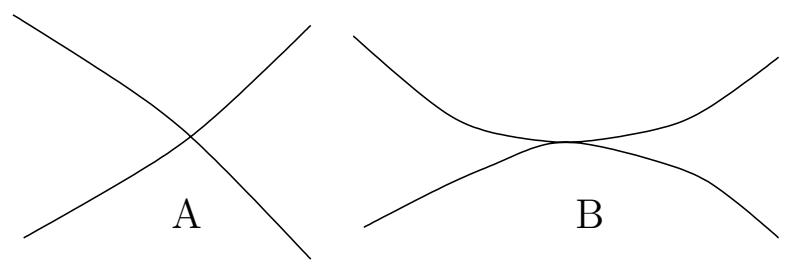

FiguRE 11.

At this point it follows easily that if $n_{x} \in \mathbb{N}, n_{x}>\frac{V(G, \lambda)}{\varepsilon}$, then there exists $y_{1} \in B_{r} \cap \Sigma^{1}$ which is not in $\mathcal{B}_{\lambda}(x)$, i.e., $\operatorname{dist}_{\Sigma}\left(x, y_{1}\right)>\lambda .\left\langle n(x), n\left(y_{1}\right)\right\rangle>0$ does not necessarily happen. Take $\bar{V} \in \mathbb{N}$ such that $\bar{V}-1<\frac{V(G, \lambda)}{\varepsilon} \leq \bar{V}$ and let $n_{\lambda}=\bar{V}^{\bar{V}}$. If $n_{x} \geq n_{\lambda}$, then there exist at least $\bar{V}$ distinct $y_{i}, i:=1, \ldots, \bar{K}$, in different components of $B_{r}(x) \cap \Sigma^{1}$ such that $\operatorname{dist}_{\Sigma}\left(x, y_{i}\right)>\lambda$. For fixed $y_{1}$ there exists $y_{2}$ among the $y_{i}$ such that $\operatorname{dist}_{\Sigma}\left(y_{1}, y_{2}\right)>\lambda$. At this point either $\left\langle n(x), n\left(y_{1}\right)\right\rangle>0$ or $\left\langle n(x), n\left(y_{2}\right)\right\rangle>0$ or $\left\langle n\left(y_{1}\right), n\left(y_{2}\right)\right\rangle>0$.

The following corollary uses Proposition 5.3 and Lemma 5.4 to obtain the upper bound on the number of graphs.

Corollary 5.5. For any $x \in \Sigma^{1}, n_{x} \leq n_{C_{l_{1}}}$.

Proof. If $n_{x}>n_{C_{l_{1}}}$, then Lemma 5.4 with $\lambda$ equal to $C_{l_{1}}$ gives that there exist $y, y^{\prime} \in B_{r}(x) \cap \Sigma^{1}$ such that $\operatorname{dist}_{\Sigma}\left(y, y^{\prime}\right)>C_{l_{1}}$ and $\left\langle n(y), n\left(y^{\prime}\right)\right\rangle>0$. Using Lemma 4.6 gives that $\mathcal{B}_{l_{1}}(x)$ is $\delta$-stable and, by Proposition [5.3. cannot be contained in $\Sigma^{1}$. $\operatorname{Since}_{\operatorname{dist}}\left(y, y^{\prime}\right)>C_{l_{1}}>l_{1}, y^{\prime}$ is not in $\Sigma^{1}$. This gives the contradiction that implies $n_{x} \leq n_{C_{l_{1}}}$.

\section{Multi-Valued Graphs in CMC surfaces}

In this final section we show that the $\Sigma_{n}^{1}$ converges $C^{2}$ to an embedded minimal disk $\Sigma_{\infty}$ that contains a multi-valued graph. It follows that the CMC surfaces in the sequence contain a multi-valued graph as well. The limit surface is embedded and minimal by a standard argument which will be sketched below. To prove that it is simply connected we need more work and well-known topological results.

Let $r>0$ be as defined in (5.2) and (5.3), that is, there exists a finite covering $B_{r}\left(x_{i}^{n}\right)$ for $\Sigma_{n}^{1}$ where everything is graphical over $T_{x_{i}^{n}} \Sigma_{n}$ and the number of graphs is uniformly bounded. We can also assume that the number of balls involved is uniformly bounded with respect to $n$. Going to a subsequence, we can assume $x_{i}^{n}$ converging to $x_{i}$ and $T_{x_{i}^{n}} \Sigma_{n}$ converging to a certain $T_{x_{i}} \Sigma_{\infty}$. Using the argument outlined in Section 2, the fact that the number of graphs is uniformly bounded, and the maximum principle for minimal surfaces gives that the limit is an embedded minimal surface. Figure 11 illustrates the two types of intersections that could occur if the limit is not embedded: A cross intersection, type A, and a tangential intersection, type B. However, type A cannot be a continuous limit of embedded surfaces. Type B, which could be the limit of a sequence of embedded surfaces, cannot occur because of the maximum principle for minimal surfaces. By continuity the curvature of this minimal surface is large at zero.

To prove that $\Sigma_{\infty}$ is simply connected we use some results about Jordan curves [10] and the following theorems. 


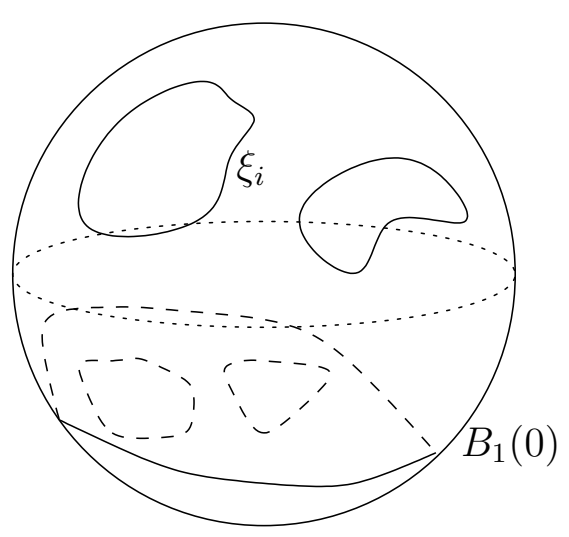

FigURE 12.

Theorem 6.1 ([10, Chapter 3]). Every compact hypersurface in Euclidean space without boundary is orientable.

Theorem 6.2 ([10, Chapter 3]). If $M$ is an orientable surface, so is $M$ minus one point.

Theorem 6.3 ([11, Corollary 3.28]). If $M$ is a closed connected n-manifold, the torsion subgroup of $H_{n-1}(M, \mathbb{Z})$ is trivial if $M$ is orientable and $\mathbb{Z}_{2}$ if $M$ is not orientable.

In our case $\Sigma_{\infty}$ is a closed connected 2-manifold, and it is a consequence of Theorem 6.3 that, if it is orientable, the torsion subgroup of its fundamental group is trivial. The next proposition shows that $\Sigma_{\infty}$ is the embedded minimal disk we have been looking for.

Proposition 6.4. $\Sigma_{\infty}$ is an embedded simply connected minimal surface such that, $0 \in \Sigma_{\infty} \subset B_{1} \subset \mathbb{R}^{3}, \partial \Sigma_{\infty} \subset \partial B_{1}, \sup _{\Sigma \cap B_{1}}|A|^{2} \leq 4 C^{2}$ and $|A|^{2}(0)=C^{2}$.

Proof. The conditions on the second fundamental form, that is,

$$
\sup _{\Sigma \cap B_{1}}|A|^{2} \leq 4 C^{2} \text { and }|A|^{2}(0)=C^{2},
$$

are a consequence of the $C^{2}$ convergence. We have already proved that it is embedded and what we are left to prove is that $\Sigma_{\infty}$ is simply connected. Let us prove that it is orientable first. We want to prove that $\Sigma_{\infty}$ is homeomorphic to a compact embedded surface minus a finite number of points. Because it is an embedded minimal surface, $\partial \Sigma_{\infty}$ is a finite number of disjoint loops $\xi_{i}, i:=1, \ldots, I$, which do not have self intersections; see Figure 12. These loops lie on $\partial B_{1}(0)$ minus one point, hence we have essentially a finite number of Jordan curves in the plane. We can glue $I$ disks to $\Sigma_{\infty}$ in a way that the result is an embedded compact surface: Each Jordan curve $\xi_{i}$ divides the plane into an inner and an outer region, and can be thought of as the boundary of a simply connected domain, namely a disk $D_{i}$. If a loop $\xi_{i}$ lies in the inside of another loop $\xi_{j}$, then we lift $D_{j}$ so that it does not intersect $D_{i}$. Figure 13 illustrates how we are gluing these disks to the surface. Since the 


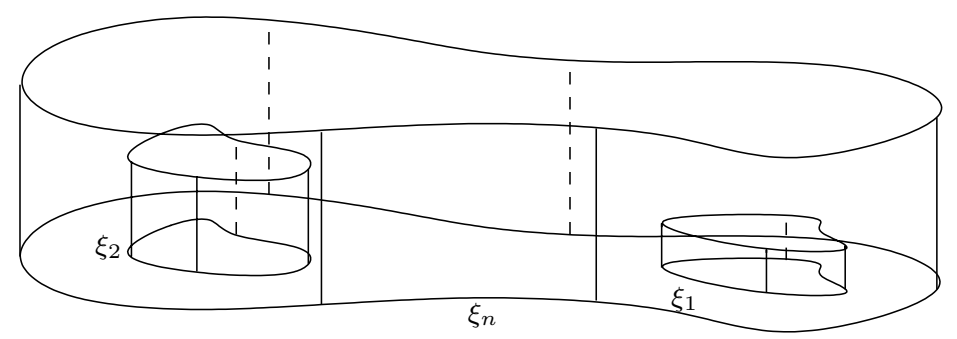

FiguRE 13.

number of loops is finite we repeat this a finite number of times and obtain in the end a new surface

$$
\bar{\Sigma}=\left\{\Sigma_{\infty} \bigcup_{i=1}^{I} D_{i}\right\} / \sim
$$

where $\sim$ is the relation that identifies $\xi_{i}$ with $\partial D_{i} . \bar{\Sigma}$ is a compact embedded surface without boundary and therefore orientable by Theorem 6.1. Theorem 6.2 implies that $\bar{\Sigma}$ takes out a finite number of points is still orientable, that is, $\bar{\Sigma} \backslash \bigcup_{i=1}^{I} D_{i}=$ $\Sigma_{\infty}$. Theorem 6.3 tells us that $\pi_{1}\left(\Sigma_{\infty}\right)$ is torsion free.

Let $\gamma: S^{1} \longrightarrow \Sigma_{\infty}$ be a closed path, and $B_{\sigma}\left(\gamma\left(t_{i}\right)\right)$ a finite covering for $\gamma$ such that $\sigma<\min (1-|\gamma|, r)$, and the number of components of $B_{\sigma}(\gamma(t))$ is nonincreasing. This is possible after going to a subsequence, assuming $n$ large because of the uniform bound. Fix a starting point $\gamma\left(t_{0}\right)$, an orientation on $\gamma$, and let

$$
\gamma_{n}\left(t_{0}\right):=\gamma\left(t_{0}\right)+s_{0} N_{\gamma\left(t_{0}\right)} \in \Sigma_{n}^{1} .
$$

Moving continuously on $\gamma(t)$ we obtain a new path,

$$
\gamma_{n}(t):=\gamma(t)+s(t) N_{\gamma(t)} \in \Sigma_{n}^{1} .
$$

The conditions on $\sigma$ and $n$ force the path to close up after it moves around $\gamma$ a finite number of times, $k \in \mathbb{N}$. Since $\Sigma_{n}^{1}$ is simply connected, there exists a map $\Gamma_{n}: D_{1} \longrightarrow \Sigma_{n}^{1}$ such that $\left.\Gamma_{n}\right|_{S^{1}}=\gamma_{n}$. Define $\Gamma: D_{1} \longrightarrow \Sigma_{\infty}$,

$$
\Gamma(x):=\lim _{n \longrightarrow \infty} \Gamma_{n}(x) .
$$

The existence of the map $\Gamma$ proves that $k \gamma$ is homotopic to a point. Since $\pi_{1}\left(\Sigma_{\infty}\right)$ is torsion free, this implies that $\gamma$ itself is homotopic to a point. Since $\gamma$ could be any path on $\Sigma_{\infty}$ we have proved that $\Sigma_{\infty}$ is simply connected.

Finally we prove that $\Sigma_{n}^{1}$ and therefore $\Sigma_{n}$ contains a multi-valued graph. In Proposition 6.4 we proved that $\Sigma_{\infty}$ is an embedded simply connected minimal surface such that,

$$
0 \in \Sigma_{\infty} \subset B_{1} \subset \mathbb{R}^{3}, \partial \Sigma_{\infty} \subset \partial B_{1}, \sup _{\Sigma \cap B_{1}}|A|^{2} \leq 4 C^{2}=4|A|^{2}(0) .
$$

Taking $C=C(N, \omega, \varepsilon)$ as in Theorem 2.1] the same theorem gives that $\Sigma_{\infty}$ contains and $N$-valued graph. Let $u$ be this $N$-valued graph, defined over $\{(\rho, \theta) \mid r \leq \rho \leq$ $s,|\theta| \leq N \pi\}$ as described in Definition 2.2. This is how we build an $N$-valued graph in $\Sigma_{n}$ : Given $r \leq \bar{\rho} \leq s$, define $u_{\bar{\rho}}(\theta)=u(\bar{\rho}, \theta)$. Consider $u_{\bar{\rho}}$ as a path on 
$\Sigma_{\infty}$ starting at $u_{\bar{\rho}}(-N \pi)$. Assuming $n$ large, $\Sigma_{n}^{1}$ moves closer and closer to $\Sigma_{\infty}$ and there exists a continuous function $\phi(\theta)$ such that

$$
u_{\bar{\rho}}^{n}(\theta)=u_{\bar{\rho}}(\theta)+\phi(\theta) \mathbf{e}_{3} \in \Sigma_{n}^{1}
$$

is well defined. The function $u^{n}(\rho, \theta)=u_{\rho}^{n}(\theta)$ defined over $\{(\rho, \theta)|r \leq \rho \leq s,| \theta \mid \leq$ $N \pi\}$ is an $N$-valued graph.

Notice that as $\Sigma_{n}^{1}$ moves closer and closer to $\Sigma_{\infty}, \Sigma_{n}^{1}$ and $\Sigma_{\infty}$ are "parallel surfaces." Not only does $\Sigma_{n}$ contain an $N$-valued graph, but the properties of this graph, such as the upper bound on the gradient, are preserved.

\section{Appendix A}

In this appendix we provide examples of CMC surfaces containing arbitrary large multi-valued graphs. We use the method of successive approximations to build a sequence of normal variations of the helicoid that converges to an embedded and simply connected CMC surface containing a multi-valued graph.

Let $\Sigma_{h}=\left\{x+u(x) N_{\Sigma}(x), x \in \Sigma\right\}$ be a normal variation of $\Sigma$, where $\Sigma$ is any minimal surface. $\Sigma_{h}$ is a CMC surface with mean curvature equal to $H$ if $u(x)$ satisfies the following equation $([12,13,15])$ :

$$
L u=H+Q(u), \quad \text { where } L u=\Delta u+|A|^{2} u,
$$

is the linearized operator. $Q$ is a quadratic and higher order function in $u, u_{i}, u_{i j}$ where $i, j \in\{1,2\}$, with geometric invariants of $\Sigma$ as coefficients. Before we prove the existence of a constant mean curvature normal variation of the helicoid we need to describe some properties of the function $Q$.

Let $C^{k, \lambda}(\Sigma)$ be the standard subset of $C^{k}(\Sigma)$ consisting of functions whose $k$ th partial derivatives are Hölder continuous with exponent $\lambda$ in $\Sigma$ and let $\|\cdot\|_{k, \lambda}$ be the notation for the Hölder norm. Let us define $\Omega_{\delta}$, the subset of $C^{2, \lambda}(\Sigma)$, in the following way:

$$
\Omega_{\delta}:=\left\{u \in C^{2, \lambda}:\|u\|_{2, \lambda}<\delta\right\} .
$$

The following lemma follows from [12, Lemma C.2] and it is a consequence of the fact that $Q$ is a quadratic and higher order function. It says that $\|Q(u)\|_{0, \lambda}$ decays faster than $\|u\|_{2}\|u\|_{2, \lambda}$.

Lemma A-1. There exist $\delta_{1}>0$ and $C_{1}>0$. So, if $u \in C^{2, \lambda}(\Sigma)$ and $|A||u|,\left|u_{i}\right|<$ $\delta_{1}$, then

$$
\|Q(u)\|_{0, \lambda}<C_{1}\|u\|_{2}\|u\|_{2, \lambda} .
$$

As a consequence of Lemma A-1, we have a new corollary that relates $\|Q(u)\|_{0, \lambda}$ and $\|u\|_{2, \lambda}$.

Corollary A-2. Given $1>C_{2}>0$ there exists $\delta_{2}>0$. So, if $u \in \Omega_{\delta_{2}}$ and $|A||u|<\delta_{2}$, then

$$
\|Q(u)\|_{0, \lambda}<C_{2}\|u\|_{2, \lambda} .
$$

Proof. Let $\delta_{2}<\min \left(\frac{C_{2}}{C_{1}}, \delta_{1}\right)$. This implies that $C_{1}\|u\|_{2}<C_{1}\|u\|_{2, \lambda}<C_{1} \delta_{2}<C_{2}$ and that $|A||u|,\left|u_{i}\right|<\delta_{2}<\delta_{1}$. Therefore, we can apply Lemma A-1 and we have

$$
\|Q(u)\|_{0, \lambda}<C_{1}\|u\|_{2}\|u\|_{2, \lambda}<C_{2}\|u\|_{2, \lambda} \text {. }
$$


Let $\Sigma$ be a simply connected disk in the helicoid that contains a multi-valued graph. Due to the domain monotonicity and continuity of eigenvalues [1] we can also assume that 0 is not an eigenvalue for $L$ on $\Sigma$ and therefore that the Dirichlet

$$
\left\{\begin{array}{l}
L u=w \\
\left.u\right|_{\partial \Sigma}=0
\end{array}\right.
$$

has a unique solution $u \in C^{2, \lambda}(\Sigma)$ for any $w \in C^{0, \lambda}(\Sigma)$. Assuming that 0 is not an eigenvalue for $L$ gives also the following lemma [9, Theorem 5.3 and page 109].

Lemma A-3. There exists a constant $B$ depending only on $\Sigma$. So, let $u \in C^{2, \lambda}(\Sigma)$ be the unique solution for (A-3). Then

$$
\|u\|_{2, \lambda}<B\|w\|_{0, \lambda} .
$$

We will prove that there exists $H>0$ such that a solution $u$ for the Dirichlet problem

$$
\left\{\begin{array}{l}
L u=H+Q(u), \\
\left.u\right|_{\partial \Sigma}=0
\end{array}\right.
$$

exists and $\|u\|_{L^{\infty}}$ is small. The existence of a fixed neighborhood of the helicoid where the normal exponential map is injective guarantees that $\Sigma_{H}=\{x+$ $\left.u(x) N_{\Sigma}(x), x \in \Sigma\right\}$ is embedded, if $\|u\|_{L^{\infty}}$ is small enough. What we are about to show is that if $H$ is small enough, we can build a sequence of normal variations $u^{n}$ of the helicoid that converges to a CMC normal variation $u$. We will also show that $\|u\|_{L^{\infty}}$ can be as small as we want and consequently that the CMC normal variation is embedded.

Let $u^{1}$ be the unique solution for

$$
\left\{\begin{array}{l}
L u^{1}=H \\
\left.u^{1}\right|_{\partial \Sigma}=0
\end{array}\right.
$$

and $u^{n}$ the unique solution for

$$
\left\{\begin{array}{l}
L u^{n}=H+Q\left(u^{n-1}\right), \\
\left.u^{n}\right|_{\partial \Sigma}=0 .
\end{array}\right.
$$

Lemma A-3 implies that

$$
\left\|u^{1}\right\|_{2, \lambda}<B H
$$

and also that

$$
\left\|u^{k}\right\|_{2, \lambda}<B\left(H+\left\|Q\left(u^{k-1}\right)\right\|_{0, \lambda}\right) .
$$

The existence of a solution for

$$
L u=H+Q(u)
$$

will follow clearly, and we will see how, applying Arzela-Ascoli to the sequence $u^{n}$ if we prove that there exists a constant $K$ such that $\left\|u^{n}\right\|_{2, \lambda}<K$ uniformly in $n$. 
Fix $C_{2}$ in Corollary A-2 so that $\varepsilon=C_{2} B<1, B$ as in Lemma A-3. We will prove by strong induction that

if $H$ is such that $B H\left(1+\frac{1}{1-\varepsilon}\right)<\delta_{2}$, then $u^{n} \in \Omega_{\delta_{2}}$ for any $n$, which is what we wanted. We already have that

$$
\left\|u^{1}\right\|_{2, \lambda}<B H<\delta_{2},
$$

namely the statement is true for $n=1$. Let us prove that

"true for $n=1$ implies true for $n=2 . "$

Assuming $\left\|u^{1}\right\|_{2, \lambda}<\delta_{2}$, we can apply Lemma $\mathrm{A}-1$ which gives that $\left\|Q\left(u^{1}\right)\right\|_{0, \lambda}<$ $C_{2}\left\|u^{1}\right\|_{2, \lambda}<\varepsilon H$; therefore,

$$
\left\|u^{2}\right\|_{2, \lambda} \leq B\left(H+\left\|Q\left(u^{1}\right)\right\|_{0, \lambda} \leq B(H+\varepsilon H)<\delta_{2} .\right.
$$

Let us prove that:

"true for all $k$ with $k \leq n$ implies true for $k=n+1$."

"True for all $k$ with $k \leq n$ " means that $\left\|u^{k}\right\|_{2, \lambda}<\delta_{2}$ for $k \leq n$ and therefore Lemma A-1 gives that

$$
\left\|Q\left(u^{k}\right)\right\|_{0, \lambda}<C_{2}\left\|u^{k}\right\|_{2, \lambda}<C_{2} B\left(H+\left\|Q\left(u^{k-1}\right)\right\|_{0, \lambda}\right)<\varepsilon\left(H+\left\|Q\left(u^{k-1}\right)\right\|_{0, \lambda}\right)
$$

for $k \leq n$. Applying $n$ times we get

$$
\begin{aligned}
\left\|u^{n+1}\right\|_{2, \lambda} & <B\left(H+\left\|Q\left(u^{n}\right)\right\|_{0, \lambda}\right)<B\left(H+\varepsilon\left(H+\left\|Q\left(u^{n-1}\right)\right\|_{0, \lambda}\right)\right) \\
& <B\left(H+\varepsilon\left(H+\varepsilon\left(H+\left\|Q\left(u^{n-2}\right)\right\|_{0, \lambda}\right)\right)\right) \\
& <B H\left(1+\sum_{k=1}^{n} \varepsilon^{k}\right)<B H\left(1+\frac{1}{1-\varepsilon}\right)<\delta_{2} .
\end{aligned}
$$

Now that we have proved that $\left\|u^{n}\right\|_{2, \lambda}<\delta_{2}$ uniformly in $n$, using Arzela-Ascoli we can extract a subsequence that converges $C^{2}$ to a certain $u \in C^{2}(\Sigma)$. Taking the limit as $n$ goes to infinity on both sides of the equation

$$
L u^{n}=H+Q\left(u^{n-1}\right)
$$

gives that

$$
L u=H+Q(u) .
$$

$u$ is therefore a constant mean curvature normal variation of the helicoid. It is clear from the proof that taking $H$ small gives $\|u\|_{L^{\infty}}$ small. Consequently, the constant mean curvature normal variation that we have built is also embedded.

\section{APPENDix B}

In this appendix we want to show that Theorem 0.1 follows from Theorem 2.3 by rescaling. The result is true even when the mean curvature is large but on a smaller ball. In other words, surfaces with large constant mean curvature have tiny multi-valued graphs around the origin. We give the idea of how that happens when dealing with simple graphs. 
Let us assume that the CMC surface $\Sigma$ is given as a graph $u$ over the unit disk (given that it contains a multi-valued graph this cannot actually happen globally, but it is always possible locally and we are assuming it now just for the sake of simplicity). Then the new surface $\Sigma^{\prime}$ given by $w(x)=R u\left(\frac{x}{R}\right)$ defined over $D_{R}(0)$ is still a CMC surface. In fact,

$$
H\left(\Sigma^{\prime}\right)=\operatorname{div}\left(\frac{\nabla w}{\sqrt{1+|\nabla w|^{2}}}\right)=\frac{1}{R} H(\Sigma) .
$$

Therefore, assuming, for instance, $R<1$ the new CMC surface has bigger mean curvature and the multi-graph happens in a smaller ball (very tiny if $H$ is big). Rescaling preserves the existence of the multi-valued graph but changes the hypotheses regarding $|A|^{2}$. Since $k_{i}^{\prime}=\frac{1}{R} k_{i}$, we have $\left|A^{\prime}\right|^{2}=\frac{|A|^{2}}{R^{2}}$ and therefore

$$
\left|A^{\prime}(0)\right|^{2}=\frac{|A(0)|^{2}}{R^{2}}=\frac{C^{2}}{R^{2}} \text { and } \sup _{\Sigma^{\prime} \cap B_{R}}\left|A^{\prime}\right|^{2}=\frac{\sup _{\Sigma \cap B_{1}}|A|^{2}}{R^{2}} \leq \frac{4}{R^{2}} C^{2},
$$

hence Theorem 0.1 .

\section{REFERENCES}

[1] I. Chavel, Eigenvalues in Riemannian Geometry, Academic Press, INC., Orlando, Florida, 1984. MR0768584 (86g:58140)

[2] T.H. Colding and W.P. Minicozzi, II, Minimal Surfaces, Courant Lecture Notes in Math., v. 4, 1999. MR 1683966 (2002b:49072)

[3] T.H. Colding and W.P. Minicozzi, The space of embedded minimal surfaces of fixed genus in a 3-manifold I; Estimates off the axis for disks, Annals of Math. 160 (2004) 27-68. MR2119717 (2006a:53004)

[4] T.H. Colding and W.P. Minicozzi, The space of embedded minimal surfaces of fixed genus in a 3-manifold II; Multi-valued graphs in a disk, Annals of Math. 160 (2004) 69-92. MR2119718 (2006a:53005)

[5] T.H. Colding and W.P. Minicozzi, The space of embedded minimal surfaces of fixed genus in a 3-manifold III; Planar domains, Annals of Math. 160 (2004) 523-572. MR2123932 (2006e:53012)

[6] T.H. Colding and W.P. Minicozzi, The space of embedded minimal surfaces of fixed genus in a 3-manifold IV; Locally simply connected, Annals of Math. 160 (2004) 573-615. MR2123933 (2006e:53013)

[7] M. do Carmo and C. K. Peng, Stable complete minimal surfaces in $\mathbb{R}^{3}$ are planes., Bull. Amer. Math. Soc. (N.S.) 1 (1979), no. 6, 903-906. MR0546314 (80j:53012)

[8] D. Fischer-Colbrie and R. Schoen, The structure of complete stable minimal surfaces in 3manifolds, Comm. Pure Appl. Math. 33 (1980) 199-211. MR0562550(81i:53044)

[9] D. Gilbarg and N. S. Trudinger, Elliptic Partial Differential Equations of Second Order, Springer-Verlag, Berlin, New York, 1983. MR0737190 (86c:35035)

[10] V. Guillemin and A. Pollack, Differential Topology, Prentice-Hall Inc., Englewood Cliffs, New Jersey, 1974. MR0348781 (50:1276)

[11] A. Hatcher, Algebraic Topology, Cambridge University Press, 2002. MR 1867354 (2002k:55001)

[12] N. Kapouleas, Complete constant mean curvature surfaces in Euclidean three-space, Ann. of Math. (2) 131 (1990), no. 2, 239-330. MR1043269 (93a:53007a)

[13] N. Kapouleas, Constant mean curvature surfaces in Euclidean spaces, Proceedings of the International Congress of Mathematicians, Vol. 1, 2 (Zürich, 1994), 481-490, Birkhäuser, Basel, 1995. MR,1403948 (97d:58053)

[14] K. Kenmotsu, Surfaces with Constant Mean Curvature, Translations of Mathematical Monographs, v. 221, AMS, 2003. MR2013507 (2004m:53014)

[15] J. C. Nitsche, Lecture on Minimal Surfaces, vol. 1, Introduction, Fundamentals, Geometry, and Basic Boundary Value Problems (English translation), Cambridge University Press, Cambridge, 1989. MR:1015936 (90m:49031) 
[16] R. Osserman, A Survey of Minimal Surfaces, Dover Publications, Inc., New York, 1986. MR0852409 (87j:53012)

[17] R. Schoen and S.-T. Yau, Lectures on Differential Geometry, Conference Proceeding and Lectures Notes in Geometry and Topology, International Press, 1994. MR.1333601 (97d:53001)

[18] S. Zhang, Curvature estimates for CMC surfaces in three dimensional manifolds, Math. Z. 249 (2005) 613-624. MR2121743 (2005i:53011)

Department of Mathematics, Johns Hopkins University, 3400 North Charles Street, 404 Krieger Hall, Baltimore, Maryland 21218-2686

E-mail address: tinaglia@math.jhu.edu

Current address: Department of Mathematics, University of Notre Dame, 255 Hurley Hall, Notre Dame, Indiana 46556-4618

E-mail address: giuseppetinaglia@nd.edu 